\title{
Direct-tuning methods for semiconductor metamaterials
}

\author{
Li Min ${ }^{1,5^{*}}$, Wenjin Wang ${ }^{1}$, Lirong Huang ${ }^{2}$, Yonghong Ling ${ }^{2}$, Tongjun Liư ${ }^{2}$ Jing Liu ${ }^{3}$, \\ Chaoming Luo $^{3}$ \& Qingdong Zeng ${ }^{4,5}$
}

Among various tunable optical devices, tunable metamaterials have exhibited their excellent ability to dynamically manipulate lights in an efficient manner. However, for unchangeable optical properties of metals, electromagnetic resonances of popular metallic metamaterials are usually tuned indirectly by varying the properties or structures of substrates around the resonant unit cells, and the tuning of metallic metamaterials has significantly low efficiency. In this paper, a direct-tuning method for semiconductor metamaterials is proposed. The resonance strength and resonance frequencies of the metamaterials can be significantly tuned by controlling free carriers' distributions in unit cells under an applied voltage. This direct-tuning method has been verified in both two-dimensional and threedimensional semiconductor metamaterials. In principle, the method allows for simplifying the structure of tunable metamaterials and opens the path to applications in ultrathin, linearly-tunable, and on-chip integrated optical components (e.g., tunable ultrathin lenses, nanoscale spatial light modulators and optical cavities with resonance modes switchable).

Numerous exotic phenomena in metamaterials (e.g., invisible cloak $^{1}$, negative refraction ${ }^{2}$, abnormal reflection ${ }^{3}$ ) have enabled a considerable number of appealing applications, such as ultra-compact wave plates ${ }^{4}$, broadband absorbers ${ }^{5}$ and optical circuit boards ${ }^{6}$. Moreover, the electromagnetic properties of metamaterials can be freely tuned by lights ${ }^{7-9}$, electrics ${ }^{10-13}$, magnetics ${ }^{14-17}$ and temperatures ${ }^{18-20}$ changing material composition or shapes of metamaterial unit cells. It is known that these optical properties are largely determined by the collective resonance movement of free electrons in metamaterial unit cells. For example, electrons' effective path length (EPL) determines the resonance wavelength, and the number of free electrons participating in resonance motions regulates the resonance strength ${ }^{21}$.

In fact, most of the reported tunable metamaterials, made of noble metals, are not likely to be tuned directly because free electrons in metallic unit cells are uncontrollable. Existing studies have focused on varying substrates' properties or structures (e.g., refractive index ${ }^{22}$, GaAs's depletion width ${ }^{23}$, graphene's optical constants ${ }^{24}$, ITO's permittivity ${ }^{25}$, PDMS's shape ${ }^{26}$ and vanadium dioxide's conductivity ${ }^{27}$ ) to tune the metamaterial response. Obviously, compared with direct metamaterial tuning, these indirect tuning methods may not excite the potential tunable ability that metamaterials deserved. On the basis of the control of free electrons in unit cells of metamaterials, direct-tuning method can lead to a sufficiently large free electron distribution and density change in a material (e.g., heavily doped semiconductor or conducting oxide), thus resulting in a large variation for resonance frequency and resonance strength of metamaterials. Predictably, directly tunable metamaterial that will be a distinctly attractive modulation approach because it not only combines advantages of individual modulation of metamaterial elements but also leads to extremely lower power dissipation.

Thus far, however, no comprehensive research has been conducted on direct tuning methods for metamaterials from directly tunable elements. Directly tunable metamaterials require that the electromagnetic property of metamaterial component is variable, and there are many similar materials. For instance, doped semiconductors (e.g., GaAs, InSb, InAs, $\mathrm{ZnO}$ ) and conducting oxide (e.g., ITO ${ }^{25}$ ) are typical materials whos free electrons (free carriers) are easy to tune by electric fields, pumping lights or temperature fields, and which can also be used to construct metamaterials ${ }^{28}$. Here, semiconductors (GaAs) were used to construct a two-dimensional (2D) and three-dimensional (3D) semiconductor metamaterials (SMs) to demonstrate a directly tunable metamaterials

\footnotetext{
${ }^{1}$ Department of Physics and Electronics, Hunan Institute of Science and Technology, Yueyang, 414000, P.R. China. ${ }^{2}$ Wuhan National Laboratory for Optoelectronics (WNLO), Huazhong University of Science and Technology, Wuhan, 430074, P.R. China. ${ }^{3}$ College of Information and Communication Engineering, Hunan Institute of Science and Technology, Yueyang, 414000, P.R. China. ${ }^{4}$ School of Physics and Electronic-information Engineering, Hubei Engineering University, Xiaogan, 432000, P.R. China. ${ }^{5}$ These authors contributed equally: Li Min and Qingdong Zeng. *email: min@hnist.edu.cn
} 
(a)

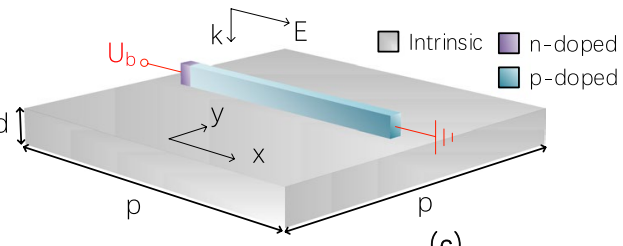

(b)

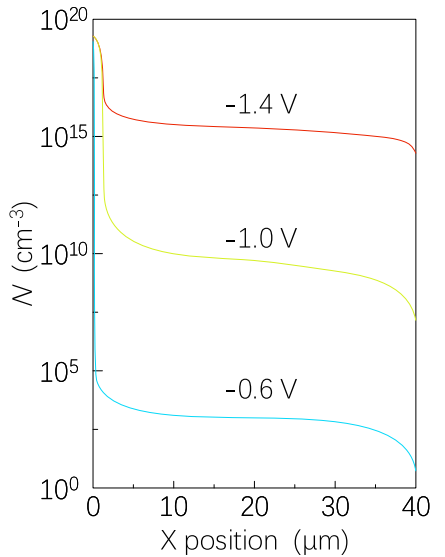

(c)

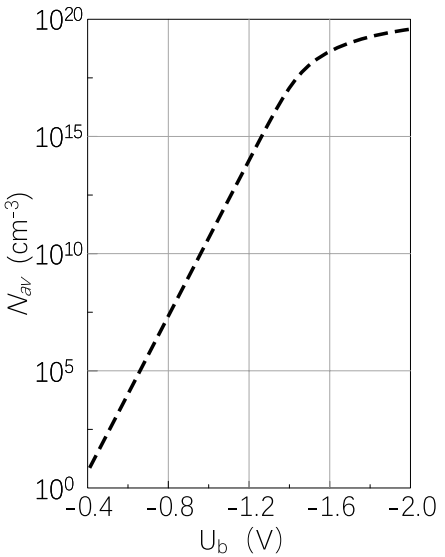

(d)

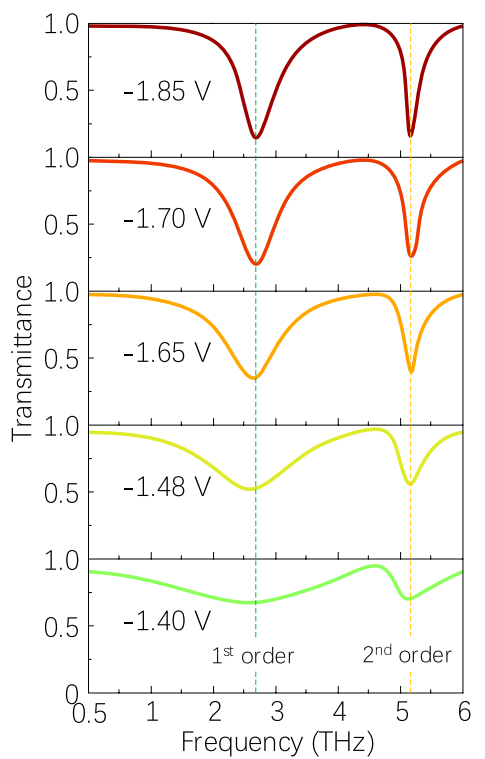

Figure 1. (a) Schematic diagram of the unit cell (strip) of 2D SMs on the substrate (intrinsic GaAs) with $\mathrm{d}=8 \mu \mathrm{m}$ and $\mathrm{p}=60 \mu \mathrm{m}$. The doped-GaAs strips have the length of $40 \mu \mathrm{m}$, the width of $4 \mu \mathrm{m}$ and the thickness of $5 \mu \mathrm{m}$. A plane wave is normally incident on the SM, with the electric field along the $\mathrm{x}$ direction. (b) Spatial distribution of the carrier concentration $N$ along the $\mathrm{x}$ direction in the strip for different applied voltages. (c) Average carrier concentration $N_{a v}$ as a function of applied bias for the exploited voltage between $-0.4 \mathrm{~V}$ and -2 V. (d) Transmittance spectra change from the 2D SM for different applied voltages. The green and yellow dash lines represent the positions of transmittance dips corresponding to the first order ( $1^{\text {st }}$ order $)$ and the second order ( $2^{\text {nd }}$ order $)$ electric resonances, respectively.

that allows dynamic electric control. In this work, unit cells of SMs are made of n-doped and p-doped semiconductors to keep the depletion region from disappearing to achieve equilibrium, and substrates are intrinsic GaAs.

\section{Results}

Directly tunable two-dimensional SMs. Two-dimensional metamaterials (also known as planar metamaterials), as a type of most popular metamaterials, are usually made of noble metals. Here, a 2D SM is presented, the unit cell of which is depicted in Fig. 1(a). A strip consists of two regions with different types of doping: an n-type region dominated by electrons, and a p-type region dominated by holes. Along the $\mathrm{x}$ direction, the $\mathrm{n}$-doped and p-doped regions in the strip have the length of $2 \mu \mathrm{m}$ and $38 \mu \mathrm{m}$ and the carrier concentration of $4.0 \times 10^{-19} \mathrm{~cm}^{-3}$ and $3.8 \times 10^{-19} \mathrm{~cm}^{-3}$, respectively. Unlike previous electrically tunable metamaterials that apply the voltages between the unit cell and the bottom substrate, the voltage bias in this work is employed on unit cells of directly tunable metamaterials. The anode contact is adjacent to the p-type region, and the cathode links to the n-type region. The voltage $U_{b}$ between the $\mathrm{n}$-doped GaAs and the $\mathrm{p}$-doped GaAs is applied, leading to the formation of free electrons accumulation in the strip. For instance, as the applied voltage $U_{b}$ increases, the depletion region narrows slowly, and increasing free carriers will gradually fill the entire p-doped region, as shown in Fig. 1(b). The average carrier concentration of $N_{a v}$ in the strip increases with the applied $U_{b}$, as shown in Fig. 1(c).

Through finite element electromagnetic simulation, the transmittance modulation of the periodically patterned antenna structure was simulated under normal incidence illumination with a transverse electric (TE) polarization (E-field along the stripes, Fig. 1(a)). As mentioned above, when the electrical bias applied on the strip is sufficiently large, free electrons will distribute widely. These free electrons will excite the first and the second order electromagnetic resonances, as shown in Fig. 1(d). In our simulations, it is assumed that when applied voltage $U_{b}$ is above $-0.4 \mathrm{~V}$, free electrons will almost evenly distribute in the strip. As a result, with the increase in gate bias, the EPL of free electrons will remain unchanged, and increasing free electrons will participate in the collective resonance motion when EM waves on the metamaterial. Figure 1(d) suggests that with the rise in the applied voltage, electric resonances (e.g., the $1^{\text {st }}$ order and $2^{\text {nd }}$ order resonances) nearly do not shift to other frequencies; they will be enhanced. It also has been verified with FDTD simulations (see Supplementary Fig. S1). Results indicate that a $\sim 42 \%$ change in the transmittance is achieved by changing the applied voltage bias from $U_{\mathrm{b}}=-1.4 \mathrm{~V}$ to $U_{\mathrm{b}}=-2 \mathrm{~V}$ at the frequency of $2.7 \mathrm{THz}$. Besides, it is noteworthy that this linear enhancement phenomenon (i.e., increasing resonance strength without varying the resonance frequencies) can be hardly found in other reported tunable metallic metamaterials and will be very suitable for linearly tunable optical components (e.g., phase retarders and linear modulators).

The EPL of free electrons in unit cells can also vary with near-field effects. It is known that the effect of near-field interactions between metallic elements in a metamaterial unit cell on the EPL cannot be neglected, especially when two subwavelength structures are very close, which can excite electromagnetically induced 

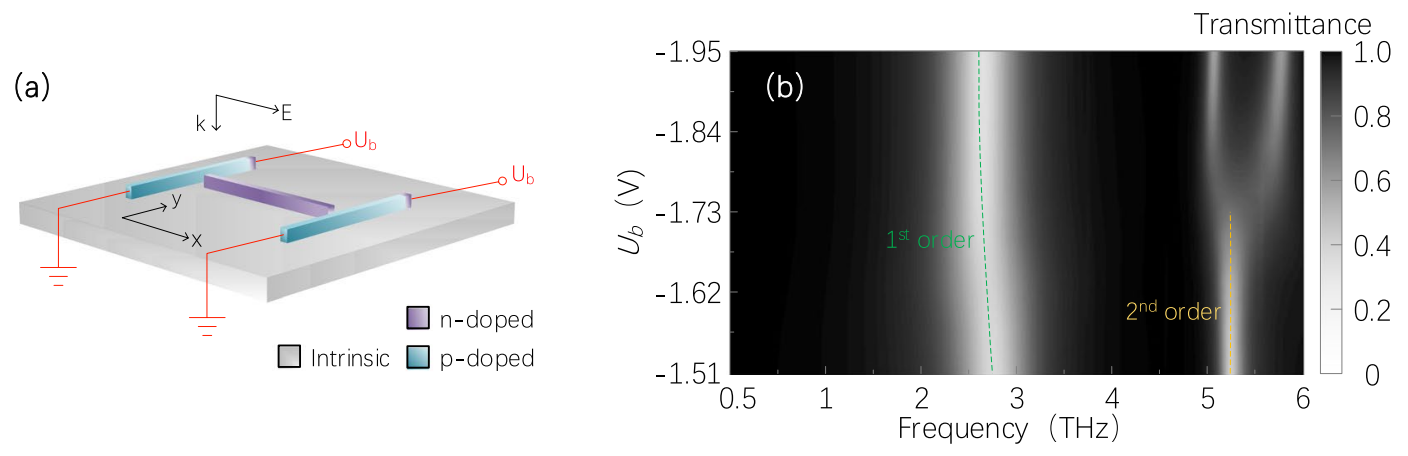

Figure 2. (a) Schematic diagram of 2D EIT SMs' unit cell consisting of three strips on the substrate (intrinsic GaAs). The geometrical parameters of the three strips are the same as the strip shown in Fig. 1(a). A plane wave is normally incident on the SM, with the electric field along the $\mathrm{x}$ direction. (b) Transmittance spectra change from the 2D EIT SM for different applied voltages $U_{b}$. The green and yellow dash lines represent the positions of transmittance dips corresponding to the $1^{\text {st }}$ order and $2^{\text {nd }}$ order electric resonances, respectively.

transparency $(\mathrm{EIT})^{29,30}$. In transparent metallic metamaterials, the EIT is usually tuned by varying the relative position of elements in unit cells ${ }^{31}$, adjusting the incident angle or polarization state of light ${ }^{32,33}$, or altering the equivalent structure of elements ${ }^{30,34}$. Here, a tunable transparent SM that enables dynamic electrical control of the EIT without changing the shape or position of elements in unit cells is numerically demonstrated. The unit cell of the transparent SM consists of a strip (made of $\mathrm{n}$-doped GaAs, carrier concentration of $\sim 5 \times 10^{19} \mathrm{~cm}^{-3}$ ) and a couple of parallel strips (both the same as that in Fig. 1(a)), as shown in Fig. 2(a).

The strip parallel to the $\mathrm{x}$ direction acts as a resonance unit cell to generate the $1^{\text {st }}$ order and $2^{\text {nd }}$ order electric resonances, in which the distribution and carrier concentration of free electrons will be affected by the near field of the other two parallel strips. According to Refs. ${ }^{31,35}$, under a normal incidence, the fundamental (i.e., the $1^{\text {st }}$ order) resonance in symmetrical-structure unit cells will not be affected seriously by the near field. In this work, the $1^{\text {st }}$ order resonance frequency of the SMs only makes a slight shift $(\sim 0.15 \mathrm{THz})$ when the applied voltage $U_{\mathrm{b}}$ rises from $-1.51 \mathrm{~V}$ to $-1.95 \mathrm{~V}$, as shown with the green dash line in Fig. 2(b).

For the $2^{\text {nd }}$ order resonance, when the applied voltage $U_{b}=0$, the two strips will have no free electrons and will not couple the incident light to form a strong near field, such that the resonance will not be affected. With the applied voltage increasing from 0 to $-1.73 \mathrm{~V}$, the coupled electric field in the two parallel strips increases in magnitude, which gradually changes the spatial distribution of free electrons in the strip, and the $2^{\text {nd }}$ order resonance strength decreases slowly at the resonance frequency of $5.2 \mathrm{THz}$, as illustrated in Fig. 2(b). When the applied voltage $>1.73 \mathrm{~V}$, the transparency window and EIT peak (between two resonance dips around the frequency of $5.2 \mathrm{THz}$ ) gradually appear. Under the applied voltage of $-1.95 \mathrm{~V}$, the original $2^{\text {nd }}$ order resonance completely disappears, and the EIT peak of $84 \%$ is observed in the transmittance spectrum, thus achieving an on-to-off EIT peak modulation. It has been further confirmed with FDTD simulations (see Supplementary Fig. S2). This physical mechanism of the EIT can be well explained by the analogy between the system and atomic EIT systems ${ }^{31,34}$.

Compared with other metallic metamaterials, field-effect modulation especially for directly tunable SMs may be a distinctly attractive approach for its wide distribution in semiconductor electronics, extremely low power dissipation and simpler modulation process (as mentioned above). It will be very suitable for on-chip integrated modulators or switchers.

Directly tunable three-dimensional SMs. Three-dimensional (3D) metamaterials (also known as bulk-like metamaterials) are another type of research interest in recent years ${ }^{36}$. Layered metamaterials, as a type of 3D metamaterials and usually composed of a sequence of dielectric layers (permittivity $\varepsilon_{d}>0$ ) and conductive layers (permittivity $\left.\varepsilon_{c}<0\right)$, can be grown with molecular beam epitaxy on lattice-matched substrates ${ }^{37}$. Moving free electrons in conductive layers can create an electric dipole, and it is critical to induce electric resonances in layered 3D metamaterials. Given this principle, noble metals are extensively taken as the conductive layers for its larger number of free electrons to stronger resonances. However, as discussed above, such metamaterials cannot be directly tuned. In fact, few tunable 3D metamaterials have been reported thus far. In this paper, a tunable 3D SM is numerically demonstrated, the resonance of which can be directly controlled with an applied voltage, as shown in Fig. 3(a).

Likewise, as the applied voltage is up-regulated (from 0 to $-1.91 \mathrm{~V}$ ), increasing free electrons diffuse into p-type region in n-p-doped layers. As a result, the increase in free carrier concentration will raise free electrons' collision rate and give rise to reduction in the EPL of free electrons. In other words, the resonance will shift to shorter wavelengths (shown with the green dashed line in Fig. 3(b)). In the meantime, it will bring about a stronger resonance. For instance, as shown in Fig. 3(b), the variation of refractive index of 3D SMs is as high as 10, which is remarkable when compared with that of naturally occurring substances. Interestingly, it can make the 3D SMs very suitable for realizing a Fabry-Perot cavity with the resonance mode switchable. For instance, at the wavelength of $20 \mu \mathrm{m}$, when the applied voltages are $U_{1}=-1.73 \mathrm{~V}, U_{2}=-1.80 \mathrm{~V}$ and $U_{3}=-1.87 \mathrm{~V}$, and corresponding refractive index of the 3D SMs are $n_{z}=2,6$ and 4, represented by the yellow, purple and red solid lines, as shown in Fig. 3(b), respectively. Also, according to the resonance condition $\left(2 k L_{y}=2 m \pi\right.$, where $k$ and $L_{y}$ are the wave number of cavity and the optical cavity's length, respectively, and $m$ is the order of resonance mode number), this $20 \mu \mathrm{m}$-wavelength light can reside in the cavity in different resonance modes, e.g., the $1^{\text {st }}, 2^{\text {nd }}$ and $3^{\text {rd }}$ order modes (see Supplementary Fig. S3). 
(a)

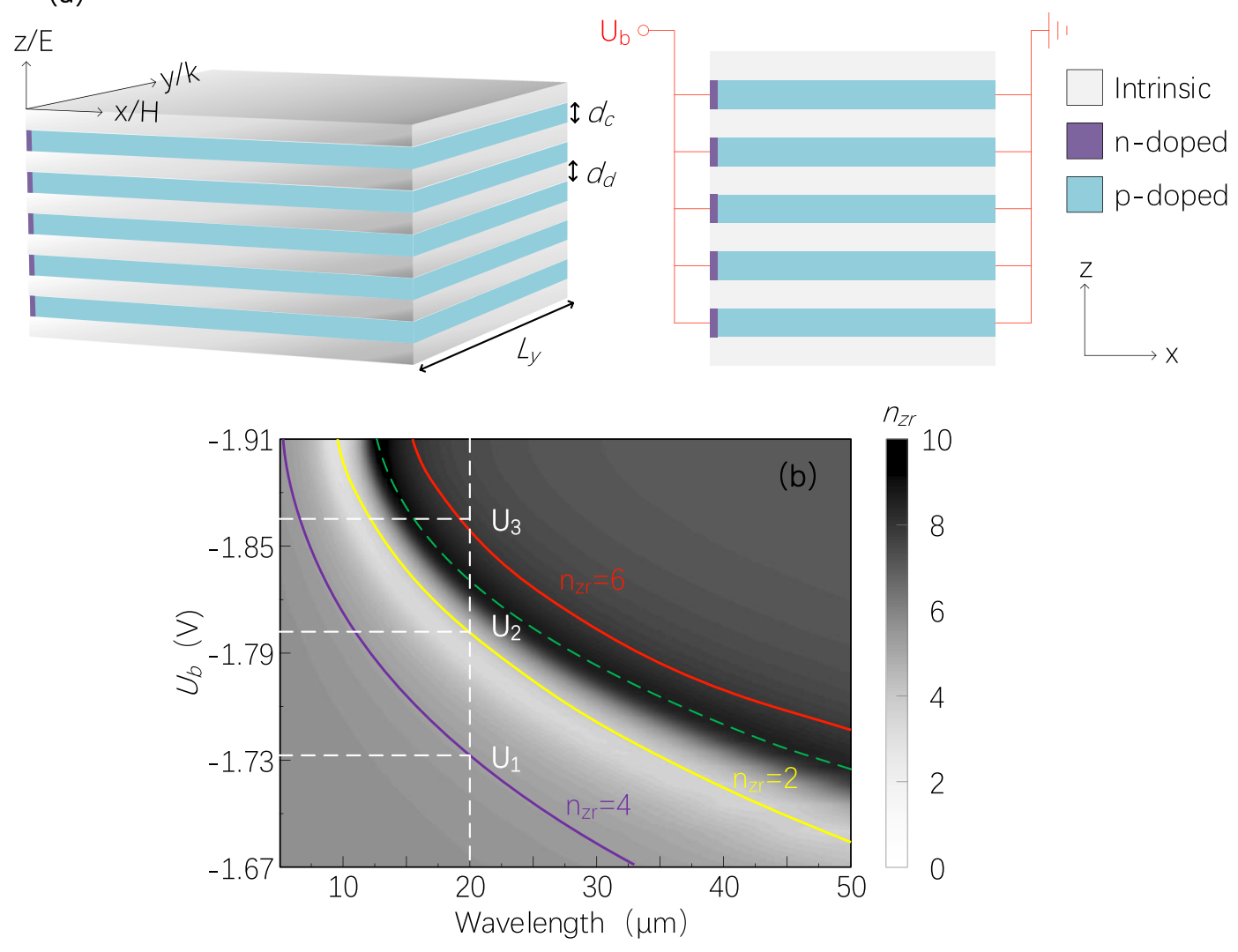

Figure 3. (a) Schematic diagram of the tunable 3D SMs with definitions of the geometrical parameters:

$L_{y}=5 \mu \mathrm{m}, d_{c}=400 \mathrm{~nm}$ and $d_{d}=400 \mathrm{~nm}$. The 3D SMs consists of 11 alternating layers of intrinsic GaAs and n-pdoped GaAs. A plane wave is incident along the y direction, with the electric field and magnetic field along the $\mathrm{z}$ direction and the $\mathrm{x}$ direction, respectively. (b) Effective refractive index $\left(n_{z}\right)$ spectra of 3D SMs in the $\mathrm{z}$ direction for different applied voltages $U_{b}$. in this study, $n_{z}=\left(\varepsilon_{z}\right)^{1 / 2}$, and $\varepsilon_{z}$ is the permittivity in the $z$ direction, expressed as $\varepsilon_{z}=\left(d_{c}+d_{d}\right) /\left(d_{c} / \varepsilon_{c}+d_{d} / \varepsilon_{d}\right)$, where $\varepsilon_{c}$ and $\varepsilon_{d}$ are permittivity of intrinsic and n-p-doped GaAs, respectively. The green dashed line represents the position of the electric resonance. The yellow, purple and red solid lines refer to locations where real parts of the refractive index $n_{z}$ are 2, 4 and 6, respectively. Dashed lines represent the corresponding values $\left(U_{2}, U_{1}\right.$ and $\left.U_{3}\right)$ of applied voltages when a light with a wavelength of $20 \mu \mathrm{m}$ leads to the formation of the $1^{\text {st }}, 2^{\text {nd }}$ and $3^{\text {rd }}$ order resonances in the Fabry-Perot cavity.

It is worthy to note that, doped semiconductors in short-wavelength bands (e.g., near-IR, visible and UV bands) cannot exhibit plasma properties (i.e., $\varepsilon_{c}>0$ ), the proposed cavity will be not available in these bands. Nevertheless, we can choose a low effective-mass and high-mobility semiconductor (e.g., indium tin oxide, zinc oxide doped with aluminum or gallium ${ }^{38}$ ) or add metallic layers in the layered SMs to alleviate this problem. Compared with previous generations of tunable metamaterials with complex structures (e.g., split-ring resonators, fishnet structure and nanoparticles), such layered SMs are easy to achieve with lithography-free thin-film deposition, which can significantly simplify the process of fabrication ${ }^{36}$.

\section{Discussion}

In conclusion, a direct-tuning method for tunable semiconductor metamaterials (SMs) is proposed, and its modulation mechanisms are reported by studying the distribution regularity of free electrons in unit cells. Results show that both the resonance frequency (or wavelength) and resonance strength of 2D and 3D SMs can be directly tuned with an applied voltage. These directly tunable SMs are promising candidates as a novel of ultra-compact and high-performance tunable optical elements and can find new possibilities for realizing integration with electronics. With the suggested methodology, other stimuli (e.g., pump lights and temperature fields) can also be used to directly tune semiconductor metamaterials. It may open a new roadmap for tunable metamaterial-based components and devices.

\section{Methods}

In the study, the numerical simulations were performed by commercial software COMSOL Multiphysics. In the simulation of free carrier distributions (e.g., spatial distribution and concentrations) in unit cells of SMs, some key physical parameters of GaAs (e.g., relative permittivity, electron mobility, hole mobility and bandgap voltage) were set as $12.9,8500 \mathrm{~cm}^{2} /(\mathrm{V} \cdot \mathrm{s}), 400 \mathrm{~cm}^{2} /(\mathrm{V} \cdot \mathrm{s})$ and $1.424 \mathrm{~V}$, respectively, in the Semiconductor Module of 
COMSOL soft. In the calculation of the permittivity of doped GaAs and the effective refractive index of SMs, relevant theories and equations in Refs. ${ }^{39-41}$ are adopted in this study. In the simulation of transmittance spectra of SMs, periodic boundary condition was set in y direction, and perfectly matched layer condition was applied in the $\mathrm{x}$ direction in the RF Module of COMSOL softs.

Received: 11 March 2019; Accepted: 8 November 2019;

Published online: 26 November 2019

\section{References}

1. Klotz, G., Mallejac, N., Guenneau, S. \& Enoch, S. Controlling frequency dispersion in electromagnetic invisibility cloaks. Scientific reports $9,6022(2019)$.

2. Kadic, M., Milton, G. W., van Hecke, M. \& Wegener, M. 3D metamaterials. Nature Reviews Physics 1 (2019).

3. Ratni, B., Lustrac, A. D., Piau, G. P. \& Burokur, S. N. Active metasurface for reconfigurable reflectors. Applied Physics A 124, 104 (2018).

4. Min, L., Huang, L., Sun, R. \& Xi, M. Dual Metamaterial With Large Birefringence. IEEE Photonics Journal 7, 1 (2015).

5. Kong, X., Xu, J., Mo, J. J. \& Liu, S. Broadband and conformal metamaterial absorber. Frontiers of Optoelectronics 10, 124 (2017).

6. Min, L. \& Huang, L. All-semiconductor metamaterial-based optical circuit board at the microscale. Journal of Applied Physics 118, 013104 (2015).

7. Dani, K. M. et al. Subpicosecond optical switching with a negative index metamaterial. Nano Letters 9, 3565 (2009).

8. Cho, D. J. et al. Ultrafast modulation of optical metamaterials. Optics Express 17, 17652 (2009).

9. Gu, J. et al. An active hybrid plasmonic metamaterial. Optical Materials Express 2, 31 (2012).

10. Anglin, K. et al. Voltage-controlled active mid-infrared plasmonic devices. Journal of Applied Physics 109, 1 (2011).

11. Karl, N. et al. An electrically driven terahertz metamaterial diffractive modulator with more than $20 \mathrm{~dB}$ of dynamic range. Applied Physics Letters 104, 091115 (2014).

12. Ratni, B., de Lustrac, A., Piau, G.-P. \& Burokur, S. N. Electronic control of linear-to-circular polarization conversion using a reconfigurable metasurface. Applied Physics Letters 111, 214101 (2017).

13. Isic, G. et al. Electrically Tunable Metal-Semiconductor-Metal Terahertz Metasurface Modulators. IEEE Journal of Selected Topics in Quantum Electronics PP, 1 (2019)

14. Han, J., Lakhtakia, A. \& Qiu, C. W. Terahertz metamaterials with semiconductor split-ring resonators for magnetostatic tunability. Optics Express 16, 14390 (2008).

15. Fu, Y. H. et al. A Micromachined Reconfigurable Metamaterial via Reconfiguration of Asymmetric Split-Ring Resonators. Advanced Functional Materials 21, 3589 (2011).

16. Jin, B. et al. Low loss and magnetic field-tunable superconducting terahertz metamaterial. Optics express 18, 17504 (2010).

17. Valente, J., Ou, J. Y., Plum, E., Youngs, I. J. \& Zheludev, N. I. A magneto-electro-optical effect in a plasmonic nanowire material. Nature Communications 6, 7021 (2015).

18. Chen, H. T. et al. Tuning the resonance in high-temperature superconducting terahertz metamaterials. Physical Review Letters 105, 247402 (2010).

19. Driscoll, T. et al. Memory metamaterials. Science 325, 1518 (2009).

20. Lewi, T., Butakov, N. A. \& Schuller, J. A. Thermal tuning capabilities of semiconductor metasurface resonators. Nanophotonics 8, 331 (2019).

21. Min, L. \& Huang, L. Perspective on resonances of metamaterials. Optics Express 23, 19022 (2015).

22. Karl, N. et al. Characterization of an active metasurface using terahertz ellipsometry. Applied Physics Letters 111, 191101 (2017).

23. Jun, Y. C. et al. Active tuning of mid-infrared metamaterials by electrical control of carrier densities. Optics Express 20, 1903 (2012).

24. Sherrott, M. C. et al. Experimental Demonstration of $>230^{\circ}$ Phase Modulation in Gate-Tunable Graphene-Gold Reconfigurable Mid-Infrared Metasurfaces. Nano Letters 17, acs.nanolett.7b00359 (2017).

25. Huang, Y. W. et al. Gate-tunable conducting oxide metasurfaces. Nano Letters 16, 5319 (2016).

26. Pryce, I. M., Aydin, K., Kelaita, Y. A., Briggs, R. M. \& Atwater, H. A. Highly strained compliant optical metamaterials with large frequency tunability. Nano Letters 10, 4222 (2010).

27. Pradhan, J. K. et al. High contrast switchability of VO2 based metamaterial absorbers with ITO ground plane. Optics Express 25, 9116 (2017).

28. Taliercio, T. \& Biagioni, P. Semiconductor infrared plasmonics. Nanophotonics (2019).

29. Liu, N. et al. Planar metamaterial analogue of electromagnetically induced transparency for plasmonic sensing. Nano Letters 10, 1103 (2010).

30. Gu, J. et al. Active control of electromagnetically induced transparency analogue in terahertz metamaterials. Nature Communications 3, 1151 (2012).

31. Na, L. et al. Plasmonic analogue of electromagnetically induced transparency at the Drude damping limit. Nature Materials 8, 758 (2009).

32. Lei, Z. et al. Polarization manipulation based on electromagnetically induced transparency-like (EIT-like) effect. Optics Express 21, 32099 (2013).

33. Jim, W. J. et al. High-performance optical retarders based on all-dielectric immersion nanogratings. Optics Letters 30, 1864 (2005).

34. Shuang, Z., Genov, D. A., Yuan, W., Ming, L. \& Xiang, Z. Plasmon-induced transparency in metamaterials. Physical Review Letters 101, $047401(2008)$

35. Xingri, J. et al. Plasmonic electromagnetically-induced transparency in symmetric structures. Optics Express 18, 13396 (2010).

36. Papadakis, G. T., Fleischman, D., Davoyan, A., Yeh, P. \& Atwater, H. A. Optical magnetism in planar metamaterial heterostructures. Nature Communications 9, 296 (2018).

37. Hoffman, A. J. et al. Negative refraction in semiconductor metamaterials. Nature Materials 6, 946 (2007).

38. West, P. R. et al. Searching for better plasmonic materials. Laser \& Photonics Reviews 4, 795 (2010).

39. Raymond, A., Robert, J. L. \& Bernard, C. The electron effective mass in heavily doped GaAs. Journal of Physics C Solid State Physics $12,2289(2001)$

40. Cardona, M. Electron effective masses of InAs and GaAs as a function of temperature and doping. Physical Review 121, 752 (1961).

41. Blakemore, J. S. Semiconducting and other major properties of gallium arsenide. Journal of Applied Physics 53, R123 (1982).

\section{Acknowledgements}

This work is supported by the National Natural Science Foundation of China (Grant Nos 61805078, 61675074, 61705064), the Natural Science Foundation of Hunan Province (Grant No. 2017JJ3098), the Natural Science Foundation of Hubei Province (Grant No. 2018CFB773), and the Foundation for Young Scientists of the Education Ministry of China (Grant No. 17B114). 


\section{Author contributions}

L.M. proposed the idea and performed numerical simulations, and prepared the manuscript. W.W. discussed the design of the proposed structure model and simulation results, improved the manuscript presentation, and supervised the whole project L.H., Y.L., T.L., J.L., C.L., Q.Z. performed all the simulations. All authors reviewed the manuscript.

\section{Competing interests}

The authors declare no competing interests.

\section{Additional information}

Supplementary information is available for this paper at https://doi.org/10.1038/s41598-019-54066-5.

Correspondence and requests for materials should be addressed to L.M.

Reprints and permissions information is available at www.nature.com/reprints.

Publisher's note Springer Nature remains neutral with regard to jurisdictional claims in published maps and institutional affiliations.

(c) (i) Open Access This article is licensed under a Creative Commons Attribution 4.0 International License, which permits use, sharing, adaptation, distribution and reproduction in any medium or format, as long as you give appropriate credit to the original author(s) and the source, provide a link to the Creative Commons license, and indicate if changes were made. The images or other third party material in this article are included in the article's Creative Commons license, unless indicated otherwise in a credit line to the material. If material is not included in the article's Creative Commons license and your intended use is not permitted by statutory regulation or exceeds the permitted use, you will need to obtain permission directly from the copyright holder. To view a copy of this license, visit http://creativecommons.org/licenses/by/4.0/.

(c) The Author(s) 2019 